\title{
A BIOMECHANICAL MODEL FOR THE ANALYSIS OF THE CERVICAL SPINE IN STATIC POSTURES
}

\author{
C. J. SNIJDERS, ${ }^{*}$ G. A. Hoek VAN DiJKe, ${ }^{*}$ and E. R. RoosCh $\dagger$ \\ *Faculty of Medicine, Department of Biomedical Physics and Technology, Erasmus University, \\ Rotterdam; and †Surgeon General's Office, Royal Netherlands Airforce, Soesterberg, The Netherlands
}

\begin{abstract}
To gain a better understanding of the forces working on the cervical spine, a spatial biomechanical computer model was developed. The first part of our research was concerned with the development of a kinematic model to establish the axes of rotation and the mutual position of the head and vertebrae with regard to flexion, extension, lateroflexion and torsion. The next step was the introduction of lines of action of muscle forces and an external load, created by gravity and accelerations in different directions, working on the centre of gravity of the head and possibly a helmet. Although the results of our calculations should be interpreted cautiously in the present stage of our research, some conclusions can be drawn with respect to different head positions. During flexion muscle forces and joint reaction forces increase, except the force between the odontoid and the ligamentum transversum atlantis. This force shows a minimum during moderate flexion. The joint reaction forces on the levels $C_{0}-C_{1}, C_{1}-C_{2}$, and $C_{7}-T_{1}$ reach minimum values during extension, each in different stages of extension. Axial rotation less than $35^{\circ}$ does not need great muscle forces, axial rotation further than $35^{\circ}$ causes muscle forces and joint reaction forces to increase fast. While performing, lateral flexion muscle forces and joint reaction forces must increase rapidly to balance the head. We obtained some indications that the order of magnitude of the calculated forces is correct.
\end{abstract}

\section{INTRODUCTION}

In high performance aircraft complicated situations arise with regard to the cervical spine under load, e.g. when sudden accelerations occur while the pilot is looking backwards. As far as the spine is concerned, most research is devoted to the lumbar area (Schulz et al., 1982a, b; Shirazi-Adl et al., 1986; Miller et al., 1986). Less research has been done on the cervical spine (Rizzi and Covelli, 1975a, b; Less and Eickelberg, 1976; Penning, 1978; Kazarian et al., 1979; Rieck, 1981; Dul et al., 1982; Glaister, 1987; McElhaney et al., 1989), which gives us little kinematic and anthropometric data. So far, interest has been focused on helmet design and helmet mounted devices (Petrofsky and Phillips, 1982; Phillips and Petrofsky, 1983). The lack of previous studies should be taken into account when considering this study. De Graef and Ingels (1982) and Aghina (1984) studied cervical complaints in the case of F-16 pilots and concluded that the origin of discomfort and fatigue was closely related to the degree and duration of the 'vertical' acceleration $\left(\boldsymbol{A}_{z}\right.$-acceleration). The weight of the helmet, the position of the head and the fatigue of the aviator also play an important role. Little attention is paid to these aspects in medical literature on aerospace. Most studies deal with the origin of acute trauma caused by unexpected movements of the aircraft and the use of an ejection seat. Experimental research has also been done on maximal sustainable forces in the neck.

A study was initiated by the Royal Netherlands Airforce Surgeon General's Office to analyse the load on neck structures under various levels of G-load. This

Received for publication 24 April 1991. paper presents the biomechanical model which was introduced to enable the calculation of the forces in a number of neck muscles and in the joints of the cervical spine. The distribution of forces in a specific joint has not been analysed. This model is used to analyse a number of flight situations, the results of these investigations will be presented in a second paper.

\section{BIOMECHANICAL MODEL}

\subsection{Kinematic model}

The head and the neck form a kinematic chain consisting of eight links. Each link has six degrees of freedom. The connecting joints restrict the degrees of freedom and the amount of motion. The upper cervical spine consists of the atlas and the axis which form a loose connection which means that muscular forces are always needed for the positioning and stability of the head. The vertebrae $C_{3}-C_{7}$ possess intervertebral discs.

The first simplification of the model is the assumption that the axes of rotation are located in the middle of the respective joints. Furthermore, the vertebrae $\mathrm{C}_{3}-\mathrm{C}_{7}$ are not modelled as separate units. The intervertebral discs and the interwoven muscles cause this part of the cervical spine to function as a unit (Penning, 1978). This allows for the vertebrae $C_{3}-C_{7}$ to be regarded as one link. The variable distance between $C_{2}$ and $C_{7}$ during bending and stretching of the cervical spine is incorporated into the model by means of a variable length of this link. Intrinsic forces in this part of the spine are not incorporated into the model. 
Figure 1 shows the model in the neutral position in the sagittal plane. Flexion/extension is possible in the points $B, C, E$ and $O$. $B$ is the centre of rotation of the atlanto occipital joint in the sagittal plane, the angle in this joint is called $\lambda . \mu$ is the angle between the atlas and the axis with $\mathrm{C}$ as the centre of rotation, $v$ is the angle between the axis and $C_{3}$ in point $E$. The origin $O$ of the coordinate system is on the caudal-dorsal corner of the vertebra $\mathrm{C}_{7}$, this is the centre of rotation of the link OE. The length of the link OE is variable, depending on the angle $\varphi$ representing the inclination of this link.

The point TC is situated at the top of the clivus, corresponding with the location of the centre of gravity of the head. PO is the point of attachment of the dorsal neck muscles at the protuberance occipitalis. The angle of the head with respect to the movement in the sagittal plane is called $\beta$. The configuration of joints in Fig. 1 represents the neutral position of the head: when the person is looking in the forward direction while his direction of view and the horizontal plane meet at an angle of $15^{\circ}, \beta$ is zero in the neutral position. Appendix A contains a complete list of all points incorporated into the model. The dimensions of parameters were found in the literature (White and Panjabi, 1978; Dul et al., 1982) or estimated on the basis of $\mathrm{X}$-ray photographs.

Figure $2 \mathrm{a}$ shows a frontal view of the model in lateroflexion, the axes of rotation for lateroflexion are shown in Fig. 2b. The axis $a_{1}$ for lateral flexion in the atlanto occipital joint is situated in point $A$, at a distance of $17 \mathrm{~mm}$ from point $\mathbf{B}$. The direction of this axis is horizontal in the neutral position. The angle between the head and the atlas is called $\theta_{1}, \theta_{2}$ is the angle between the axis and $C_{3}$, point $E$ is the centre of lateral flexion at this level. The axis of rotation $a_{2}$ is the bisector of the angle OED. The link OE is able to

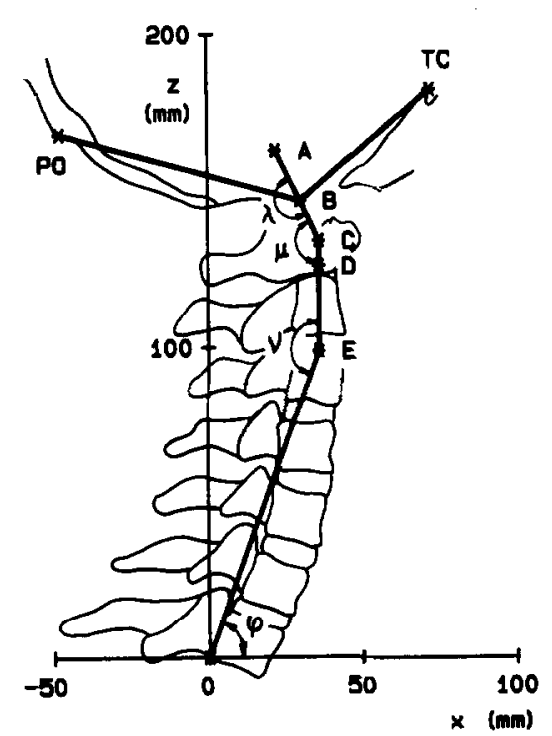

Fig. 1. Side-view of the kinematic model, neutral position. Ventral side is at the right.
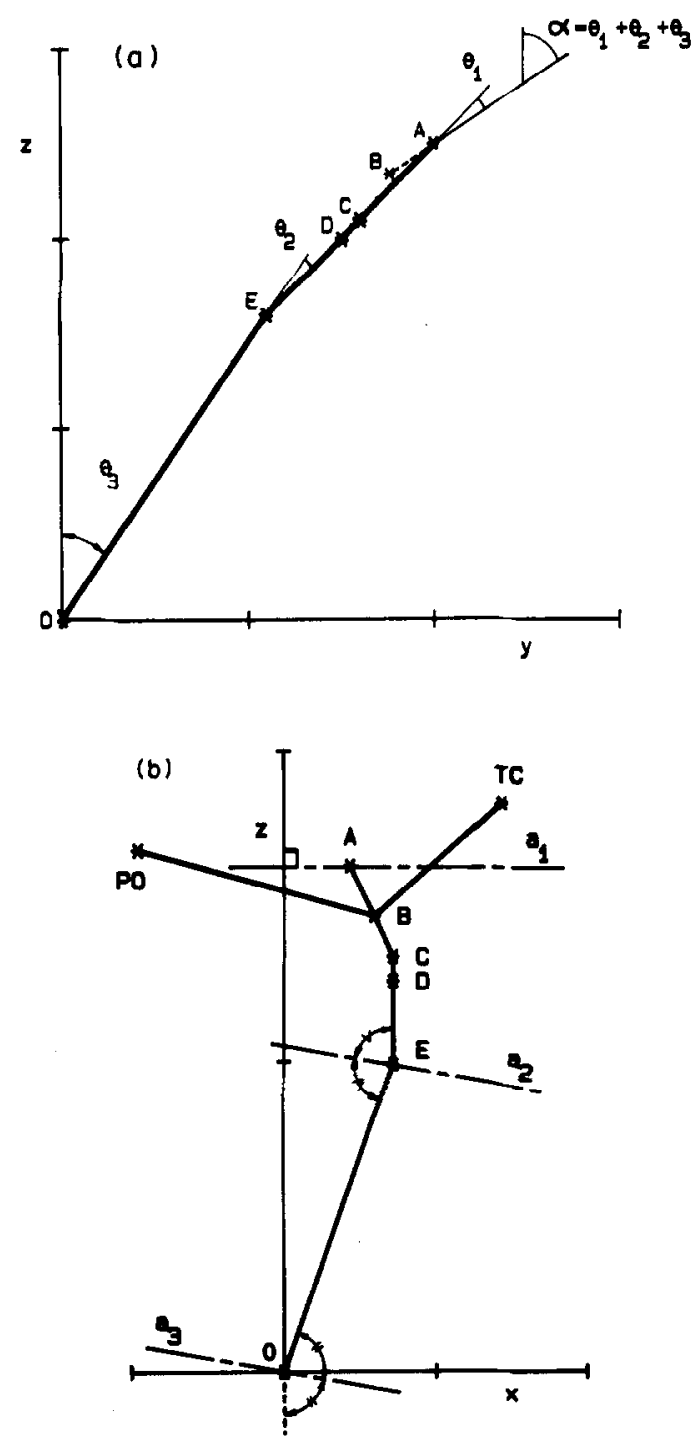

Fig. 2. (a) Front view of the kinematic model in lateroflexion (graph not to scale). (b) Axes of rotation for lateroflexion.

perform lateral flexion on point $O$ on an axis $a_{3}$ which coincides with the bisector of the angle between $\mathrm{OE}$ and the negative part of the $z$-axis. The angle of this rotation is called $\theta_{3}$. No lateral flexion is assumed to take place on $C_{1}-C_{2}$. The angle of the head with respect to the movement in the frontal plane is called $\alpha$.

The axial rotation $\gamma$ of the head is the result of the contributions $\rho_{1}$ on the level $C_{1}-C_{2}$ and $\rho_{2}$, spread over $C_{3}-C_{7}$. The centre of rotation for $\rho_{1}$ is the odontoid, represented by point $D, 8 \mathrm{~mm}$ below point $C$. The spread rotation over $C_{3}-C_{7}$ is represented as a single rotation on $\mathrm{OE}$.

The next part of the kinematic model deals with the relations between the head and the respective vertebrae. The location of points in the neutral position and the relations for forward flexion are based on anthropometric data valid for an average adult man 
(Dul et al., 1982). For other movements the relations are based on the proportions of the limits of motion of the respective joints (White and Panjabi, 1978).

2.1.1. Forward flexion. A distinction is made between the stages knicking in $\mathrm{C}_{0}-\mathrm{C}_{1}$ during the first $8^{\circ}$ of rotation, and buckling and bending of $\mathrm{C}_{1}-\mathrm{C}_{2}$, $C_{2}-C_{3}$ and $C_{3}-C_{7}$ for angles between 8 and $45^{\circ}$. In this phase $C_{0}-C_{1}$ shows a relative retroflexion from +8 to $-8^{\circ}$. So for these stages of flexion the following algorithms were assumed in the kinematic model:

$$
\begin{aligned}
0 \leqslant \beta \leqslant 8^{\circ}: \quad \Delta \lambda=\beta \\
\Delta \mu=\Delta v=\Delta \varphi=0, \\
8 \leqslant \beta \leqslant 45^{\circ}: \quad \Delta \lambda=8^{\circ}-(16 / 37) *(\beta-8)^{\circ} \\
\Delta \mu=(8 / 37) *\left(\beta-8^{\circ}\right) \\
\Delta v=(20 / 37) *(\beta-8)^{\circ} \\
\Delta \varphi=-(25 / 37) *(\beta-8)^{\circ} .
\end{aligned}
$$

(N.B.: The angles $\lambda, \mu, v$ and $\varphi$ have initial values which describe the neutral position, shown in Fig. 1. In this position the angles $\alpha, \beta$ and $\gamma$ are zero. The angles $\Delta \lambda$, $\Delta \mu, \Delta v$ and $\Delta \varphi$ are changes with respect to the neutral position.) Due to the decreasing lordosis of the cervical spine while rotating the head forward, the distance between $\mathrm{O}$ and $\mathrm{E}$ increases. In the literature (Dul et al., 1982), the length of the link $O E$ was found only for $0 \leqslant \beta \leqslant 8^{\circ}\left(O E_{1}\right)$ and for $\beta=45^{\circ}\left(O E_{2}\right)$. The length $O E$ of this link is calculated by linear interpolation between these values:

$0 \leqslant \beta \leqslant 8^{\circ}: \quad \mathrm{OE}=\mathrm{OE}_{1}$

$8 \leqslant \beta \leqslant 45^{\circ}: \mathrm{OE}=\mathrm{OE}_{1}+\frac{\left(\mathrm{OE}_{2}-\mathrm{OE}_{1}\right)}{37} *(\beta-8)^{\circ}$.

\subsubsection{Backward flexion.}

$$
\begin{aligned}
-89^{\circ} \leqslant \beta \leqslant 0^{\circ}: \Delta \lambda & =(13 / 89) * \beta \\
\Delta \mu & =(10 / 89) * \beta \\
\Delta v & =(33 / 89) * \beta \\
\Delta \varphi & =(33 / 89) * \beta .
\end{aligned}
$$

No data are available about the distance between the points $\mathbf{O}$ and $E$, so the same relationship as for forward flexion is assumed:

$$
\mathrm{OE}=\mathrm{OE}_{1}+\frac{\left(\mathrm{OE}_{2}-\mathrm{OE}_{1}\right)}{37} *(\beta)^{\circ} .
$$

No substantial influence is expected by the simplification of linear interpolation between $\beta=8$ and $45^{\circ}$, because the lengthening of $\mathrm{OE}$ concerns only $5 \mathrm{~mm}$ from $105 \mathrm{~mm}\left(O E_{1}\right)$ to $110 \mathrm{~mm}\left(O E_{2}\right)$. Additional research is needed to validate the algorithm for $\beta \leqslant 0^{\circ}$.

2.1.3. Lateral flexion. The relations between $\theta_{1}, \theta_{2}$ and $\theta_{3}$ are given by:

$$
\begin{aligned}
& \theta_{1}=(8 / 55) * \alpha \\
& \theta_{2}=(10 / 55) * \alpha \\
& \theta_{3}=(37 / 55) * \alpha .
\end{aligned}
$$

A positive value of $\alpha$ refers to lateroflexion to the left. 2.1.4. Axial rotation. The angles $\rho_{1}$ and $\rho_{2}$ are computed as follows:

$$
\begin{aligned}
& \rho_{1}=(47 / 98) * \gamma \\
& \rho_{2}=(51 / 98) * \gamma .
\end{aligned}
$$

A positive value of $\beta$ refers to axial rotation to the left.

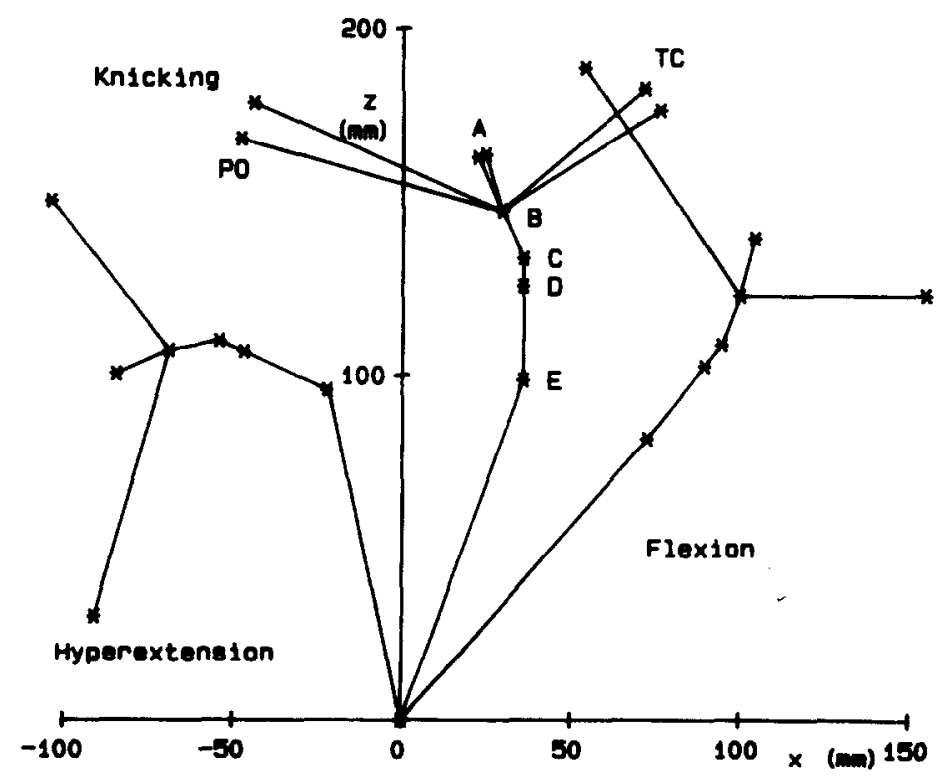

Fig. 3. Different stages of flexion/extension movement: hyperextension, knicking of the head and forward flexion. The first $8^{\circ}$ from neutral position, the head knicks in $C_{01}$. The next phase between 8 and $45^{\circ}$ knicking of the head, buckling of the atlas and bending of the lower cervical spine occur. 


\subsection{Muscle forces}

The next step in biomechanical modelling was the introduction of the forces produced by the muscles. First, the muscles were selected that are supposed to make the largest contribution to the stabilization of the head and the neck. This selection was made on the basis of size and moment arm of the various muscles. The muscles which are incorporated into the model are listed in Appendix A. Next, the origin and insertion of each muscle had to be estimated, based on anatomy text books and anthropometric literature (Kiss and Szentágothai, 1964; von Lanz and Wachsmuth, 1955; Wells, 1971; Romanes, 1981).

Figure 4 shows a side view, a frontal view and a three-dimensional drawing of the link $\mathrm{C}_{0}$. FTRL and
FTRR are the lines of action of the left and right trapezius muscle, respectively. The letters FSCML and FSCMR are short for left and right sternocleidomastoid muscle. FRC is the $m$. rectus capitus represented here by one line of action attached to the frontal side of the arc of the atlas, point $\mathrm{C} 1 \mathrm{~V}$. The origin is located on the pars basilaris, point PB.

The input parameters for the model are the weight of the head and, if required, acceleration forces and the weight of a helmet. No friction is assumed in the joints, so the joint reaction force consists of three components (in $x$-, $y$ - and $z$-directions). Basically, three muscle forces are sufficient to equilibrate the six degrees of freedom of the head. Due to the number of muscle forces (five muscle forces for the link $\mathrm{C}_{0}$ ), the static (a)

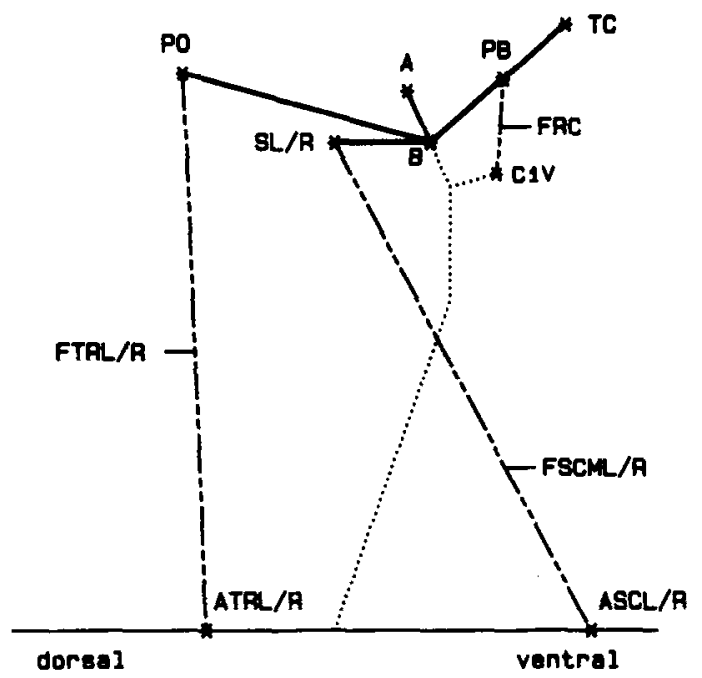

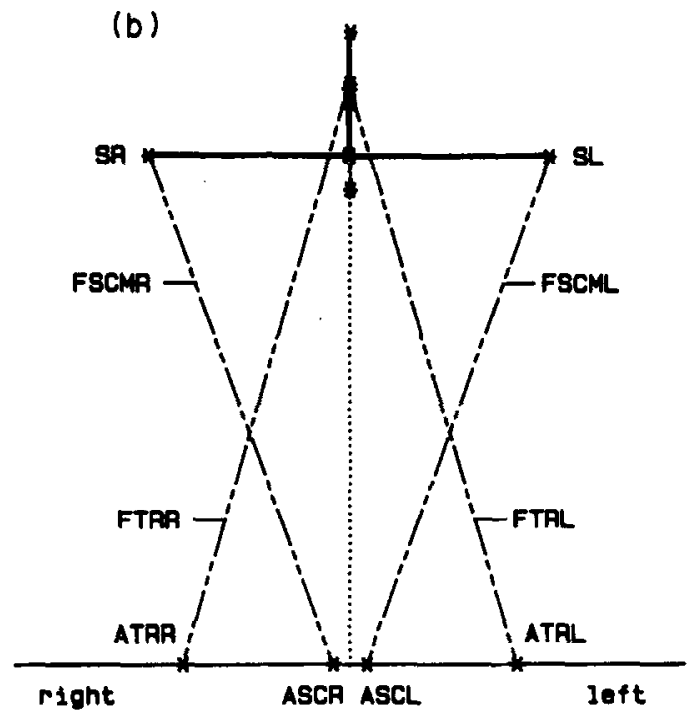

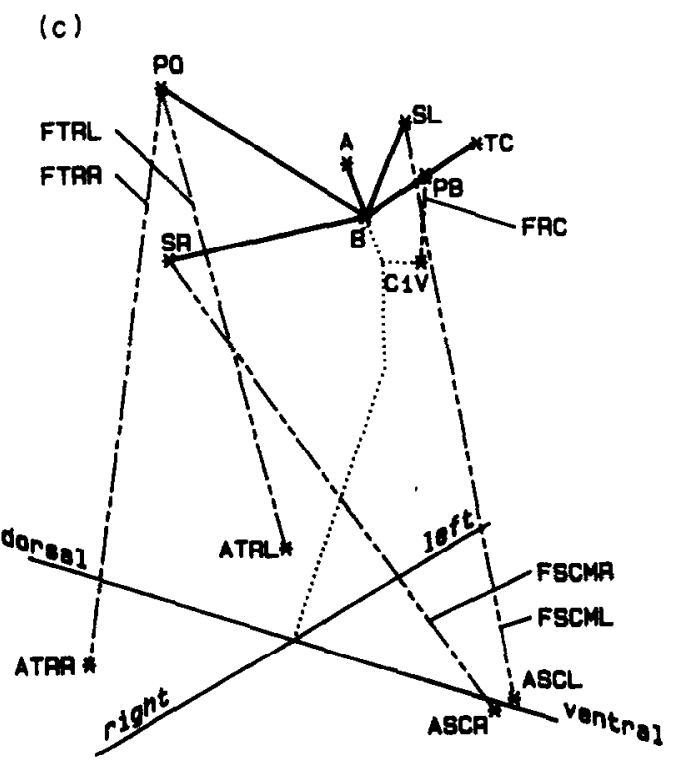

Fig. 4. Side view (a), front view (b) and three-dimensional drawing (c) of the link $\mathrm{C}_{0}$. The dashed lines represent the lines of action of the muscles acting on this link. 
model is overdetermined. Therefore, a basic optimization algorithm was used which calculates the muscle forces and joint reaction force for every combination of three muscles. Considering the restriction that muscles only can produce tension forces, the solution which led to the smallest joint reaction force in the atlanto occipital joint was chosen as the final solution (Schultz et al., 1982a, b). The choice for this criterion will be discussed later. From the calculation of the equilibrium of the head, the muscle forces and the total reaction force in the atlanto occipital joint were derived.

Next, the same procedure was followed to calculate the equilibrium of the atlas, with the atlanto occipital joint reaction force calculated above as an input parameter. The reaction force in the $\mathrm{C}_{2}-\mathrm{C}_{3}$ joint was input parameter for a similar procedure for the lower cervical spine. With regard to the equilibrium of the atlas, special attention was paid to the force in the ligamentum transversum atlantis. As shown in Fig. 5 , the force between odontoid and ligament (FT) can become substantial when the person is bending forward.

The force and moment balance equations are presented in Appendix B.

\section{RESULTS}

With the help of the model muscle forces and joint reaction forces can be estimated. Figure 6 is the result of the calculation of the equilibrium of moments and

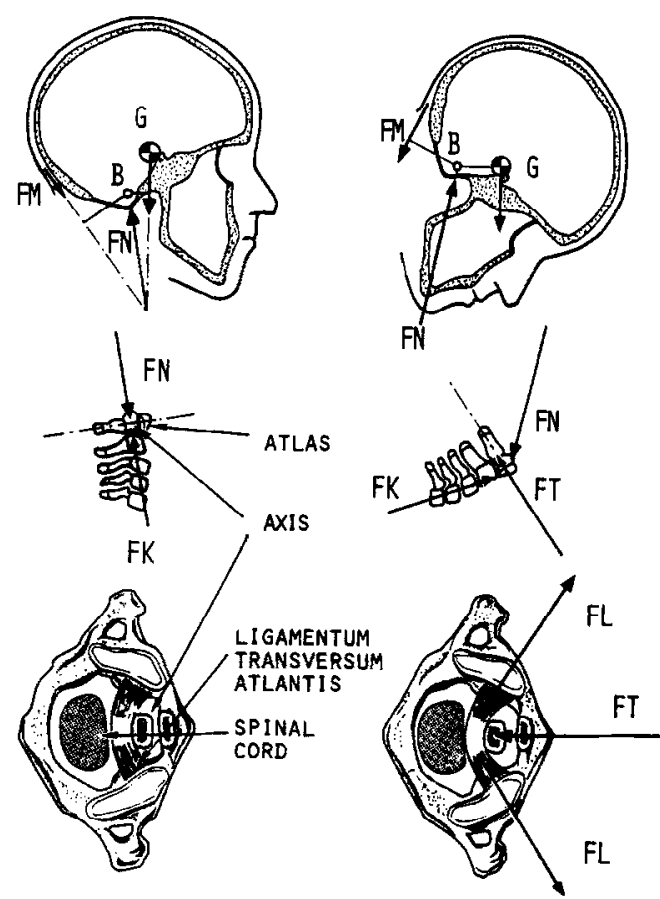

Fig. 5. Force FT in the ligamentum transversum atlantis arising from pressure of the odontoid, preventing shearing of the atlas on the axis. forces when the head is in the neutral position. Here, the only input parameter is the weight of the head, being $45 \mathrm{~N}$ (Williams and Lissner, 1962). In this position no muscle force is needed to equilibrate the atlas.

From the neutral position, calculations can be made for flexion, extension, lateroflexion, torsion and combinations of these different rotations. In Fig. 7a the joint reaction forces are shown as a function of flexion and extension, Fig. $7 \mathrm{~b}$ shows the muscle forces. The muscle forces not shown in the graph are zero. For $\beta=$ $-55^{\circ}$ the centre of gravity of the head is above the atlanto occipital joint. In this situation no muscle force is needed to equilibriate the head, the joint reaction force $\mathrm{FN}$ reaches a minimum value. A similar situation occurs for $\beta=-30^{\circ}$ for the link $C_{3}-C_{7}$ : at this angle the load on this link is pointed at point $O$. During flexion and extension no muscle force is needed to balance the atlas. The forces $F K$ and $F T$ are sufficient to equilibriate the load $F N$.

The muscle forces, as predicted by the model, are just minimum forces to balance the links. There will also be muscle force for reasons of stability, this will be discussed later.

In Fig. 8a the joint reaction forces are calculated for the maximal excursion in axial rotation. Figure $8 \mathrm{~b}$ shows the muscle forces acting on $\mathrm{C}_{0}$, Fig. $8 \mathrm{c}$ the muscle forces on $\mathrm{C}_{1}$ and Fig. $8 \mathrm{~d}$ the muscle forces acting on $\mathrm{C}_{3}-\mathrm{C}_{7}$. While rotating the head, the centre of gravity stays at the ventral side of the atlanto occipital joint, so FRC is not necessary for the equilibrium of the head.

The graphs show a great difference between rotations less than $35^{\circ}$ and rotations greater than $35^{\circ}$. Rotating the head less than $35^{\circ}$ in a positive direction

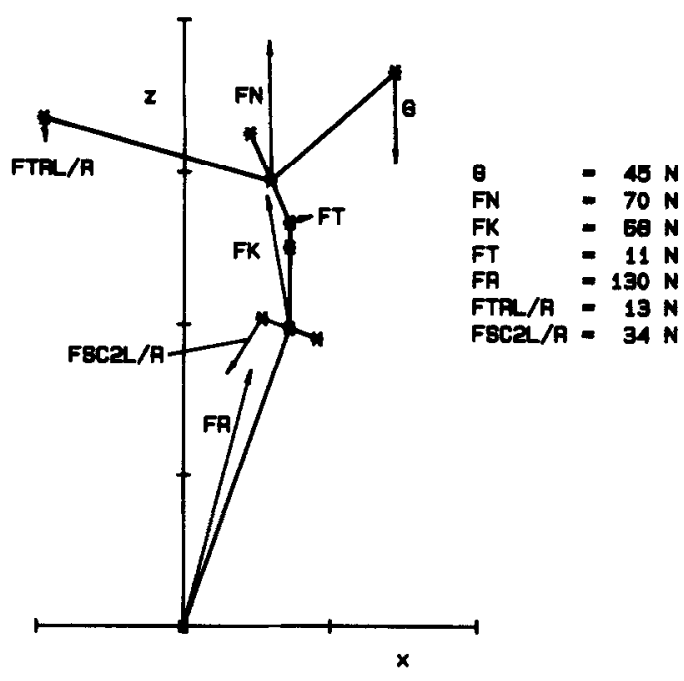

Fig. 6. Forces in muscles and joints when the head is in the neutral position. FN is the joint reaction force in the atlanto occipital joint. FK in $C_{2}-C_{3}$ and $F R$ in $C_{7}-T_{1}$. The forces are drawn acting from a caudal structure (muscle or condyle) on a cranial structure. The joint reaction forces are in the sagittal plane whereas the muscle forces are not. 

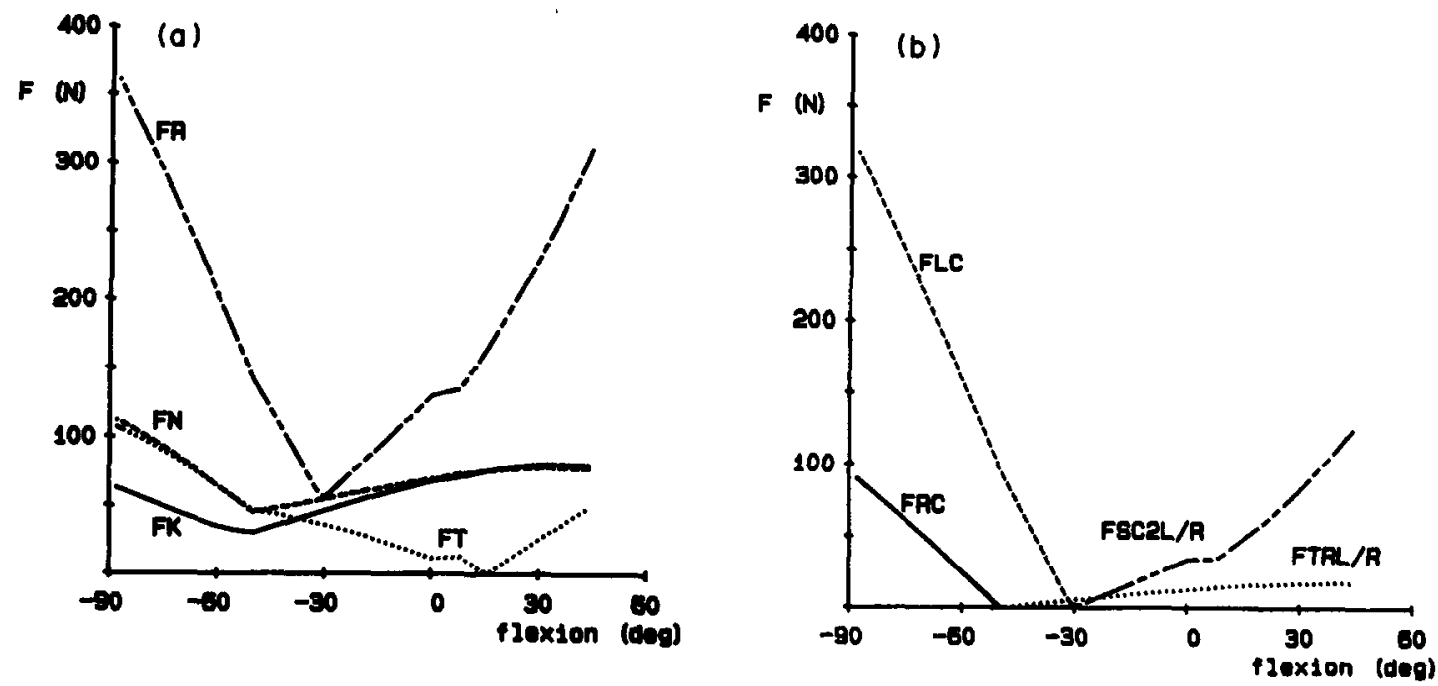

Fig. 7a, b. Joint reaction forces and muscle forces as a function of flexion ( $\beta$ ).
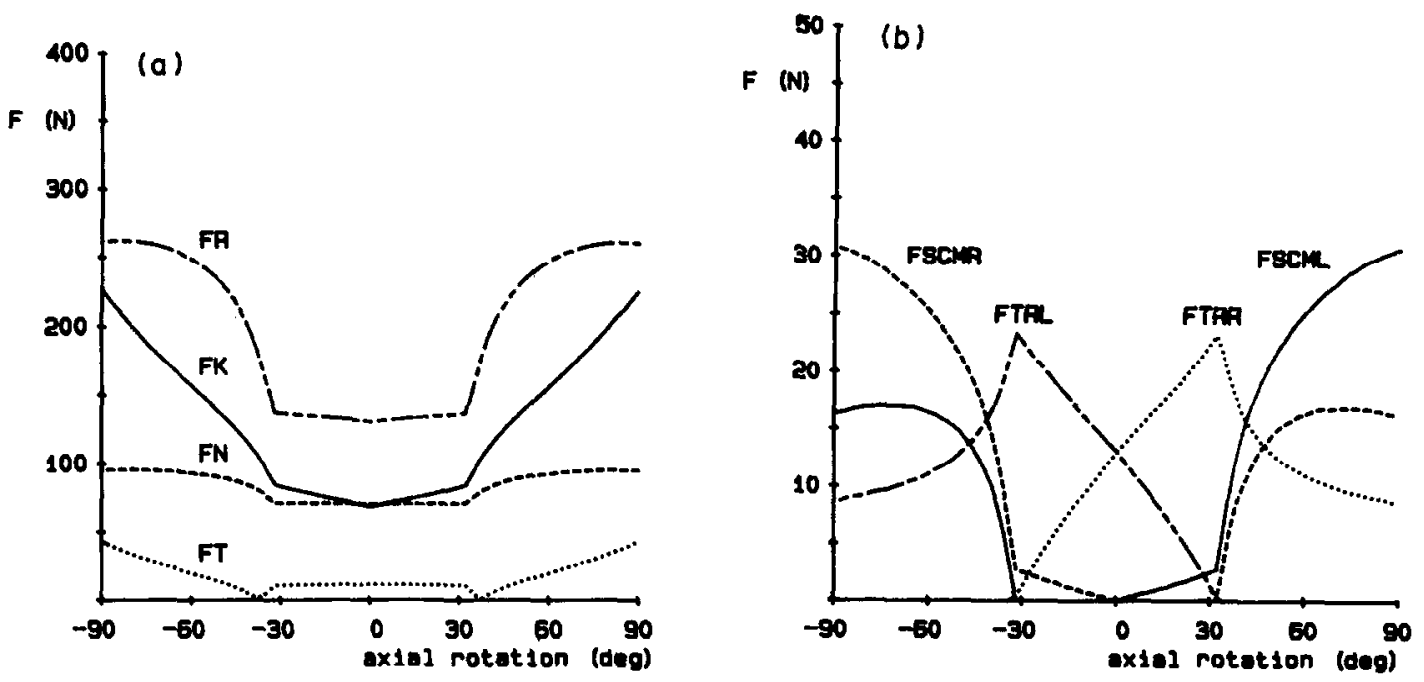

(d)
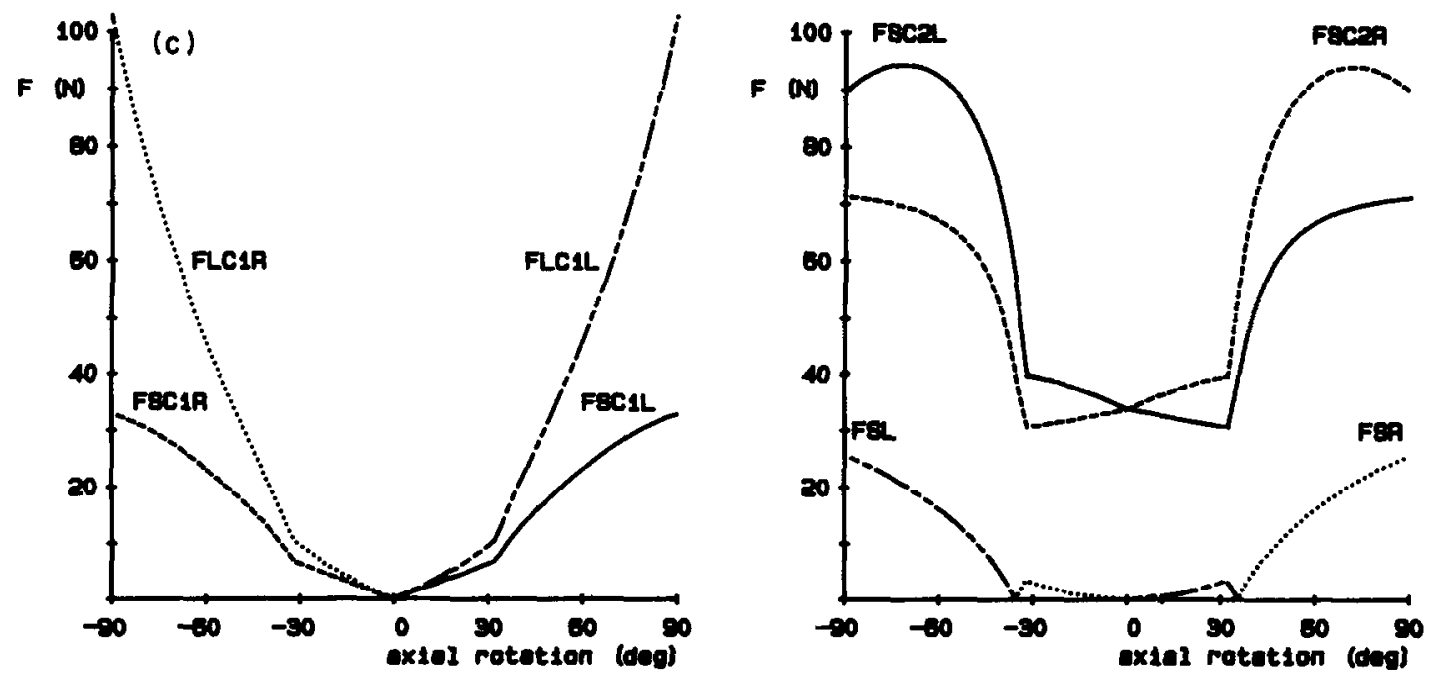

Fig. 8a, b, c, d. Joint reaction forces and muscle forces as a function of axial rotation ( $y$ ). 
(to the left) is possible by increasing muscle force FTRR and decreasing force FTRL, see Fig. 9. Their sum is almost constant and in the vertical direction. The point of attachment of the $\mathrm{m}$. trapezius $\mathrm{PO}$ moves to the right. Only little force of the sternocleidomastoid muscle is needed to balance the head. When $\gamma=35^{\circ}$ the line of action of FTRR is almost vertical and further rotation is not possible only by altering FTRL and FTRR. For greater rotations the right sternocleidomastoid muscle is needed. However, the direction of the line of action of this muscle is very unfavourable to achieve axial rotation of the head. Action of the left sternocleidomastoid muscle is needed to balance the head in the frontal plane, causing the joint reaction forces to increase fast. Besides, the $\mathrm{m}$. trapezius, which is necessary to equilibriate the head in the sagittal plane, gives a moment which opposes further rotation, see Fig. 9.

Similar to Fig. 8, Fig. 10 shows the joint reaction forces and muscle forces during lateral flexion. As expected, the $\mathrm{m}$. sternocleidomastoideus must be active. Due to the dorsal position of SL and SR with respect to point B (see Fig. 4a), the $\mathrm{m}$. sternocleidomastoideus also gives a moment on the $y$-axis. This moment relieves the $\mathrm{m}$. trapezius. For lateroflexion of more than $16^{\circ}$ this moment becomes so great that action of $\mathrm{m}$. rectus capitus is needed to prevent the head from rotating backward.

\section{DISCUSSION}

Before drawing conclusions, it must be emphasized that the model is a simplification of reality and that reliable anthropometric data hardly exist. Some calculated forces can be compared with data in the literature. Rizzi and Covelli (1975a, b) calculated the force of the $m$. trapezius in the neutral position and, in

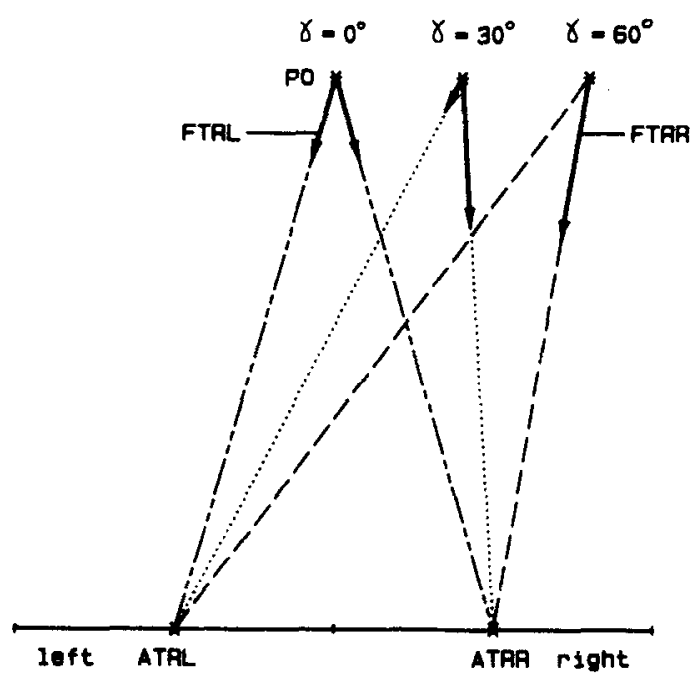

Fig. 9. Back view on the m. trapezius. Distribution of muscle force over left and right trapezius muscle during various stages of axial rotation (graph not to scale). extreme flexion, they found for the sum of the left and right muscle 19 and $39 \mathrm{~N}$. Less and Eickelberg (1976) estimated the neck muscle force for two positions between neutral position and extreme flexion as 24 and $41 \mathrm{~N}$. The forces calculated by the model are 26 and $36 \mathrm{~N}$, these values are of the same order of magnitude.

An indication of a correct proportion of forces follows from considering the joint reaction forces on the levels $C_{2}-C_{3}$ and $C_{7}-T_{1}$ in relation to the area of the intervertebral discs. The ratio of these areas is circa $1: 2.5$, which is consonant to the ratio of the forces FK:FR. The mean ratio in various positions of the head is $1: 2.4$, see Table 1 .

A sensitivity analysis was performed to determine which parameters affected the results the most. In the neutral position and in the extreme positions all parameters were varied one by one up to a deviation of $10 \%$. A parameter was considered to be critical when its influence on the results was more than $10 \%$. As expected, the geometric data in the neutral position turned out to be the most critical. Varying the parameter $\varphi_{0}$ caused the joint reaction force FR to deviate as much as $25 \%$. This same force showed a deviation of $60 \%$ when all parameters were given a deviation of $10 \%$. So the model turned out to be rather sensitive for geometric data, emphasizing the importance of reliable anthropometric data.

The optimization algorithm leads to the minimal joint reaction forces. Synergistic muscle forces and muscle forces for stabilization are not incorporated into the model. Due to the fact that in vivo measurements of muscle forces are impossible, the choice for an optimization criterion only can be made by intuitively reasonable assumptions. The field of interest for this study comcerns F-16 flight situations where neck loads occur which cause vertebrae to fail. Especially in these situations, the criterion of least possible joint reaction force seems reasonable. Both muscle action for stabilization and other strategies to obtain equilibrium will increase these reaction forces. The estimation of joint reaction forces by this model will be a conservative one, in reality the joint reaction forces may be greater. A number of optimization techniques for knee flexion was compared by Dul et al. (1984).

Table 1. Ratio between FK and FR in different situations, spread over the range of motion of $\alpha, \beta$ and $\gamma$

\begin{tabular}{lrrc}
\hline & FK & FR & FK:FR \\
\hline Neutral & 68 & 130 & $1: 1.9$ \\
$\alpha=25^{\circ}$ & 178 & 274 & $1: 1.5$ \\
$\alpha=50^{\circ}$ & 262 & 402 & $1: 1.5$ \\
$\beta=40^{\circ}$ & 80 & 287 & $1: 3.6$ \\
$\beta=-40^{\circ}$ & 38 & 98 & $1: 2.6$ \\
$\beta=-80^{\circ}$ & 54 & 315 & $1: 5.8$ \\
$\gamma=25^{\circ}$ & 80 & 136 & $1: 1.7$ \\
$\gamma=50^{\circ}$ & 136 & 231 & $1: 1.7$ \\
$\gamma=75^{\circ}$ & 260 & 260 & $1: 1.4$ \\
\hline
\end{tabular}



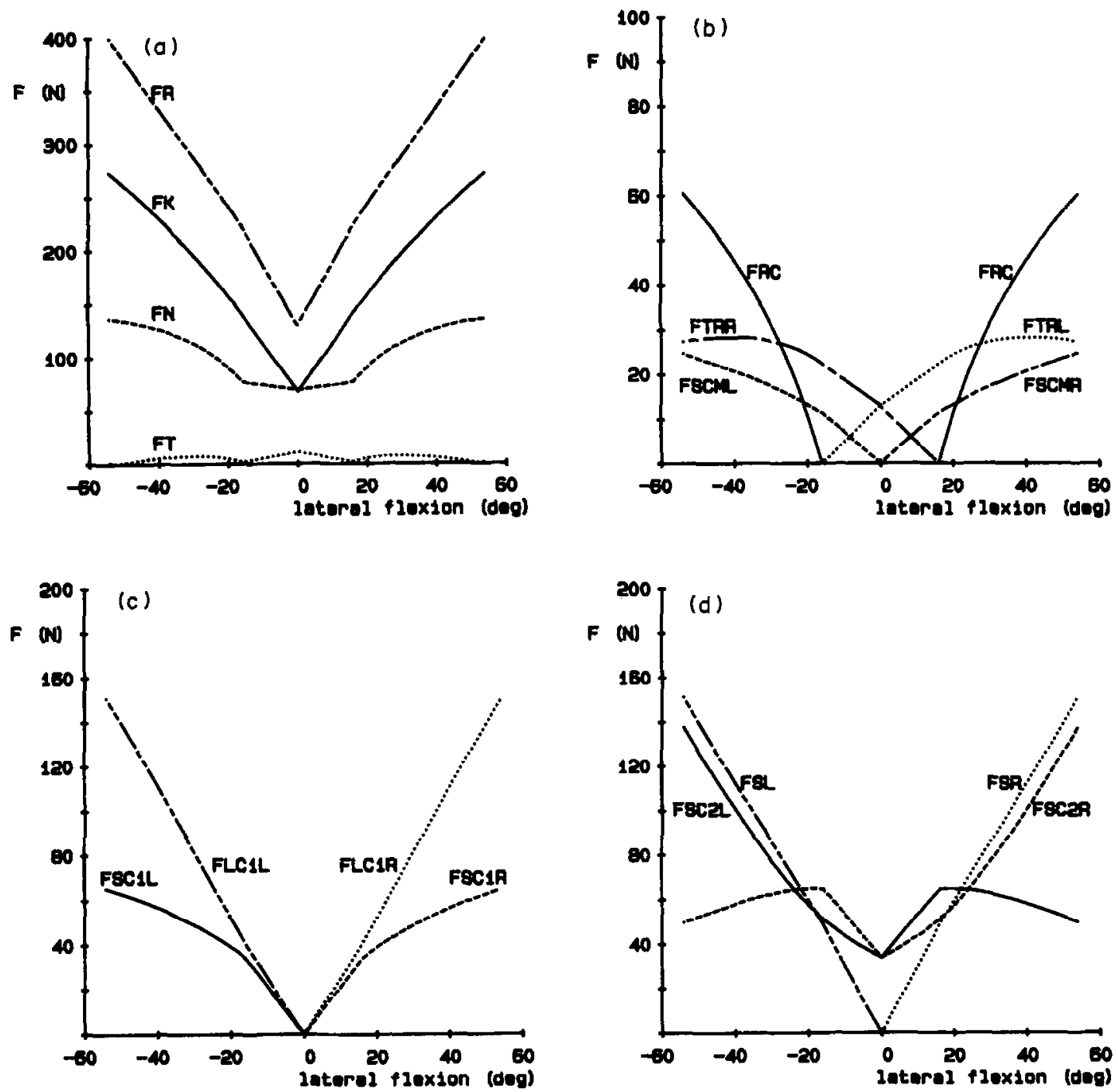

Fig. 10a, b, c, d. Joint reaction forces and muscle forces as a function of lateral flexion $(\alpha)$.

\section{CONCLUSIONS}

With the help of the described neck model, muscle forces and joint reaction forces in the neck can be estimated in various situations. Although it is difficult to verify the model as far as demonstrating real existing forces is concerned, we obtained indications that the order of magnitude is correct. So the approach followed leads to results, be it of limited accuracy. The most reliable conclusions can be drawn with respect to comparing different situations.

During flexion, especially, the joint reaction force on $C_{7}-T_{1}$ and the force on ligamentum transversum atlantis increase. The joint reaction forces are minimal in a situation of backward flexion ( $30^{\circ}$ for FR, $55^{\circ}$ for FN and FK). From a biomechanical point of view this is a favourable situation, but not comfortable for physiological reasons. In normal situations the trapezius muscle is active, therefore this muscle must be relatively strong. During axial rotation of less than $35^{\circ}$, only small changes in muscle force and joint reaction force appear. From $35^{\circ}$ these forces increase very fast. A possible example of this fact can be seen by people in a meeting who move their chair when they have to look aside for a long time.

Muscle forces and joint reaction forces (except FT) also increase rapidly during lateral flexion, making these situations uncomfortable. However, in daily life, situations in which people are forced into lateral flexion for a sustained amount of time are not common.

\section{REFERENCES}

Aghina, J. C. F. M. (1984) Cervicale klachten bij jachtvliegers. IGDKLu, Soesterberg (internal report, in Dutch).

Dul, J., Snijders, C. J. and Timmerman, P. (1982) Bewegungen und Kräfte im oberen Kopfkelenk beim Vorbeugen der Halswirbelsäule. Manuelle Medizin 20, 51-58. 
Dul, J., Townsend, M. A., Shiavi, R. and Johnson, G. E. (1984) Muscular synergism-I. On criteria for load sharing between synergistic muscles. J. Biomechanics 17, 663-667.

Glaister, D. H. (1987) The effects of off-axis loading on head mobility. Proceedings Air Crew Helmets and Helmet Mounted Devices, Royal Aeronautical Society, London, $10 \mathrm{Feb}$, 13.1-13.4. Hauts GU 14-6SZ, U.K.

Graef, P. de and Ingels, M. (1982) Het optreden van cervicalgien bij piloten onderworpen aan langdurig aangehouden positieve versnellingen. Acta. Med. Mil. Belg. 134, 115-119.

Kazarian, L. E., Belk, W. F. and Hoffman, H. P. (1979) Traumatic lesions of the cervical spine 1971-1979. Agardograph/AMRL-TR-80-53.

Kiss, F. and Szentagothai, J. (1964) Atlas of Human Anatomy, Vol. 1. Pergamon Press, Oxford.

Lanz, T. von and Wachsmuth, W. (1955) Praktische Anatomy (Teil Hals). Springer, Berlin.

Less, M. and Eickelberg, W. W. B. (1976) Force changes in neck vertebrae and muscles. In Biomechanics $V \cdot A$ (Edited by Komi, P. V.). University Park Press, Baltimore.

McElhaney, J. H., Doherty, B. J., Paver, J. G., Myers, B. S and Grey, L. (1989) Flexion, extension and lateral bending responses of the cervical spine. Proceedings 67 th Aerospace Medical Panel Meeting, Agard, Munchen.

Miller, J., Schultz, A., Warwick, D and Spencer, D. (1986) Mechanical properties of lumbar spine motion segments under large loads. J. Biomechanics 19, 79-84.

Penning L. (1978) Normal movements of the cervical spine. Am. J. Roentgenol. 130, 317-326.

Petrofsky, J. S. and Phillips, C. A. (1982) The strengthendurance relationship in skeletal muscle: its application to helmet design. Aviation Space Envir. Med. 53, 365-369.

Phillips, C. A. and Petrofsky, J. S. (1983) Neck muscle loading and fatigue: systematic variation of headgear weight and centre-of-gravity. Aviation Space Envir. Med. 54, 901-905.

Rieck, A. (1981) To the fundamentals of the isometric muscle training as a physical counteraction of the jet pilot against 'G'-loads. Air Crew Safety and Survivability, AGARD, 1-6.

Rizzi, M. A. and Covelli, B. (1975a) Die Funktion der Nackenmuskulatur biomechanisch berechnet. Manuelle Medizin 13, 101-106.

Rizzi, M. A. and Covelli, B. (1975b) Biomechanischer Beitrag zur Berechnung der Kräfte der Halsmuskulatur und deren Wirkung. Z. Orthop. 113, 371-377.

Romanes, G. J. (1981) Cunningham's Textbook of Anatomy. Oxford University Press, Oxford.

Schulz, A. B., Andersson, G. B. J., Haderspeck, K., Örtengren, R., Nordin, M. and Björk, R. (1982b) Analysis and measurements of lumbar trunk loads in tasks involving bends and twists. $J$. Biomechanics, 15, 669-675.
Schulz, A., Andersson, G., Örtengren, R., Haderspeck, K. and Nachemson, A. (1982a) Loads on the lumbar spine: validation of a biomechanical analysis by measurements of intradiscal pressures and myoelectric signals. J. Bone $J t$ Surg. 64, 713-720.

Shirazi-Adl, S. A., Ahmed, A. M. and Shrivastava, S. C. (1986) A finite-element study of a lumbar motion segment subjected to pure sagittal plane moments. J. Biomechanics 19, $331-350$.

Wells, K. F. (1971) Kinesiology. W. B. Saunders, Philadelphia.

White, A. A. and Panjabi, M. M. (1978) Clinical Biomechanics of the Spine. J. B. Lippincott, Philadelphia.

Williams, M. and Lissner, H. R. (1962) Biomechanics of Human Motion. W. B. Saunders, Philadelphia.

\section{APPENDIX A}

List of points in model:

E centre of rotation $\mathrm{C}_{2}-\mathrm{C}_{3}$ flcxion-extension and lateral flexion

D centre of axial rotation $\mathrm{C}_{1}-\mathrm{C}_{2}$

C centre of rotation $C_{1}-C_{2}$ flexion-extension

B centre of rotation $\mathrm{C}_{0}-\mathrm{C}_{1}$ flexion-extension

A centre of rotation $\mathrm{C}_{0}-\mathrm{C}_{1}$ lateral flexion

TC top clivus, centre of gravity head

Table 2. Position of points in neutral position

\begin{tabular}{lrrrlrrr}
\hline Point & $x$ & $\begin{array}{c}y \\
(\mathrm{~mm})\end{array}$ & & Point & $x$ & $\begin{array}{c}y \\
(\mathrm{~mm})\end{array}$ & $z$ \\
\hline E & 35 & 0 & 98 & C2VL & 45 & 17 & 94 \\
D & 35 & 0 & 125 & C2VR & 45 & -17 & 94 \\
C & 35 & 0 & 133 & C2VM & 45 & 0 & 94 \\
B & 29 & 0 & 146 & C2DL & 26 & 20 & 101 \\
A & 22 & 0 & 162 & C2DR & 26 & -20 & 101 \\
TC & 71 & 0 & 162 & & & & \\
PB & 52 & 0 & 166 & ATRL & -40 & 50 & 0 \\
PO & -48 & 0 & 167 & ATRR & -40 & -50 & 0 \\
SL & -0.8 & 60 & 146 & ASCL & 80 & 5 & 0 \\
SR & -0.8 & -60 & 146 & ASCR & 80 & -5 & 0 \\
& & & & ALC & 15 & 0 & 0 \\
C1V & 50 & 0 & 137 & ASPL & -40 & 0 & 0 \\
C1M & 45 & 0 & 135 & ASPR & -40 & 0 & 0 \\
C1DL & 16 & 5 & 127 & ASL & 15 & 75 & 0 \\
C1DR & 16 & -5 & 127 & ASR & 15 & -75 & 0 \\
\hline
\end{tabular}

Table 3. List of muscles incorporated into the model

\begin{tabular}{lll}
\hline Abbreviation & $\begin{array}{c}\text { Points of } \\
\text { attachment }\end{array}$ & \multicolumn{1}{c}{ Name of muscle } \\
\hline FTRL & $\begin{array}{l}\text { PO-ATRL } \\
\text { PO-ATRR }\end{array}$ & $\begin{array}{l}\text { m. trapezius left } \\
\text { FTRR }\end{array}$ \\
FSCM & SL-ASCL & m. sternocleidomastoid left \\
FSCMR & SR-ASCR & m. sternocleidomastoid right \\
FSC1L & C1DL-C2DL & m. splenius cervicis left \\
FC1R & C1DR-C2DR & m. splenius cervicis right \\
FLC1L & C1V-C2VL & m. longus colli left \\
FLC1R & C1V-C2VR & m. longus colli right \\
FSC2L & C2DL-ASPL & m. splenius cervicis left \\
FSC2R & C2DR-ASPR & m. splenius cervicis right \\
FLC & C2VM-ALC & m. longus colli \\
FSL & C2DL-ASL & m. scaleni left \\
FSR & C2DR-ASR & m. scaleni right \\
\hline
\end{tabular}


PB pars basilaris

PO protuberance occipitalis

SL proc. mastoideus left

SR proc. mastoideus right

CiV ventral side atlas

C1M massa lateralis atlas

CIDL dorsal side atlas left

CIDR dorsal side atlas right

C2VL ventral side $C_{2}$ left (tuberculum anterius)

C2VR ventral side $\mathrm{C}_{2}$ right

C2VM ventral side $\mathrm{C}_{2}$ middle

$\mathrm{C} 2 \mathrm{DL}$ dorsal side $\mathrm{C}_{2}$ left

C2DR dorsal side $\mathrm{C}_{2}$ right

ATRL intersection in $x-y$ plane line of action m. trapezius left

ATRR intersection in $x-y$ plane line of action m. trapezius right

ASCL intersection in $x-y$ plane line of action $m$. sternocleidomastoideus left

ASCR intersection in $x-y$ plane line of action $\mathrm{m}$. sternocleidomastoideus right

ALC intersection in $x-y$ plane line of action $\mathrm{m}$. longus colli

ASPL intersection in $x-y$ plane line of action $\mathrm{m}$. splenius cervicis left

ASPR intersection in $x-y$ plane line of action $\mathrm{m}$. splenius cervicis right

ASL intersection in $x-y$ plane line of action $\mathrm{m}$. scaleni left

ASR

intersection in $x-y$ plane line of action $\mathrm{m}$. scaleni right

\section{APPENDIX B}

In this appendix the calculation of the equilibrium a fictitious link is given in an arbitrary position (see Fig. A1). The points $D, L$ and $R$ are defined as points of attachment of muscles on this link. The lines of action for the muscles are drawn as dashed lines. The points DD, LL and RR are the intersections of the lines of action in the $x-y$ plane. Point $M$ is the centre of rotation.

The input parameter is a known force FG with components $F G_{x}, F G_{y}$ and $F G_{z}$ acting on point $V$. The moment of this force on point $M$ is given by the equation:

$$
\mathbf{M G}=\mathbf{F G} \times(\mathbf{V}-\mathbf{M}) \text {. }
$$

This moment vector has three components, their magnitudes are called $M G_{x}, M G_{y}$ and $M G_{z}$. The joint reaction force $F M$, which has three independent components $F M_{x}, F M_{y}$ and $F M_{2}$, acts on point $M$, so it has no moment on this point.

The direction of lines of action of the muscles are defined by D-DD, L-LL and R-RR. The muscle force vector F1, corresponding with the first line of action, can be written as:

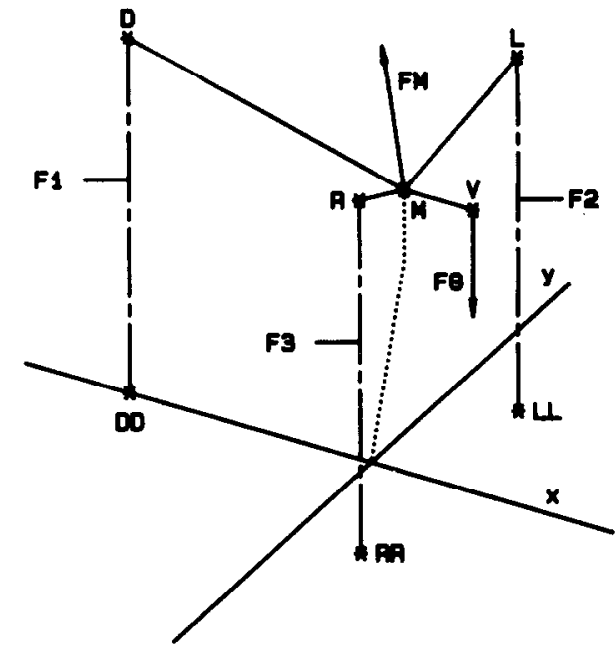

Fig. A1. A fictitious link in equilibrium with one joint, three muscle forces and an external load.

force. This unit vector can be written as DU, so equation (A2) can be rewritten as:

$$
F 1=K_{1} * \text { DU }
$$

In the same way the muscle forces $F 2$ and $F 3$ can be defined as:

$$
\begin{aligned}
& F 2=K_{2} * L U \\
& F 3=K_{3} * R U .
\end{aligned}
$$

The moment M1 of F1 on point $M$ can be written as:

$$
\mathbf{M 1}=\mathbf{F 1} \times(\mathbf{D}-\mathbf{M}) \text {, }
$$

or, using equation (A3):

$$
\mathrm{M1}=K_{1} * \mathrm{DU} \times(\mathrm{D}-\mathrm{M}) \text {. }
$$

Equation (A7) can be abbreviated to:

$$
\text { M1 }=K_{1} * \text { DP. }
$$

In the same way the moments $\mathrm{M} 2$ and $\mathrm{M3}$ can be written as:

$$
\begin{aligned}
& \mathrm{M} 2=K_{2} * \mathrm{LP}, \\
& \mathrm{M3}=K_{3} * \mathrm{RP} .
\end{aligned}
$$

The equations which describe the equilibrium are:

$$
\begin{array}{r}
\mathbf{F} 1+\mathbf{F} 2+F 3+F_{x}+F_{y}+F_{z}=-F G \\
M 1+M 2+M 3=-M G,
\end{array}
$$

or, in matrix notation:

$$
\left[\begin{array}{llllll}
D U_{x} & L U_{x} & R U_{x} & 1 & 0 & 0 \\
D U_{y} & L U_{y} & R U_{y} & 0 & 1 & 0 \\
D U_{z} & L U_{z} & R U_{z} & 0 & 0 & 1 \\
D P_{x} & L P_{x} & R P_{x} & 0 & 0 & 0 \\
D P_{y} & L P_{y} & R P_{y} & 0 & 0 & 0 \\
D P_{z} & L P_{z} & R P_{z} & 0 & 0 & 0
\end{array}\right] *\left[\begin{array}{l}
K_{1} \\
K_{2} \\
K_{3} \\
F M_{x} \\
F M_{y} \\
F M_{z}
\end{array}\right]=\left[\begin{array}{l}
-F G_{x} \\
-F G_{y} \\
-F G_{z} \\
-M G_{x} \\
-M G_{y} \\
-M G_{z}
\end{array}\right]
$$

$$
F 1=K_{1} * \frac{(D-D D)}{|D-D D|} .
$$

$K_{1}$ is a scalar, representing the magnitude of this muscle force, the quotient is a unit vector in the direction of this
The matrix is filled with geometrical data, the right term represents the load. The muscle forces $K_{1}, K_{2}, K_{3}$ and the components of the joint reaction force can be determined by solving this system. 